\title{
Problemdisparitäten Talgebiet - Berggebiet und agrarpolitische Möglichkeiten zu deren Abbau
}

\section{Einleitung}

Der Titel dieses kurzen Beitrages erfordert, daß einerseits auf Disparitäten im innerlandwirtschaftlichen Bereich eingetreten wird. Dies wird also eine Situationsanalyse sein. Solche Analysen - wenn auch in unterschiedlicher Tiefe - sind oft anzutreffen. Viel schwieriger sind anderseits konsistente und überzeugende Darstellungen von agrarpolitischen Möglichkeiten zu finden, die zum Abbau dieser Disparitäten führen. Wie wir zeigen werden, gibt es zwar solche Möglichkeiten; wenn sie nicht oder viel zu spät angewendet werden, so mag der Grund hiefür in der praktischen Politik liegen. Dies soll uns jedoch nicht hindern, stets nach neuen Lösungsansätzen zu suchen und auftretende neue Probleme frühzeitig zu erkennen. Manche Anstöße zu Neuerungen in der Agrarpolitik der Schweiz in den letzten dreißig Jahren kamen aus der Wissenschaft.

\section{Disparitäten und ihre Ursachen}

Von den vielen möglichen Disparitäten zwischen Regionen sind aus wirtschaftlicher Sicht vor allem Disparitäten in den Einkommen, in der Arbeitsbelastung, in der sozialen Anerkennung und (soweit erfaßbar) in der Sinngebung durch die Arbeit zu beachten. Zweifellos bestehen zwischen diesen vier genannten möglichen Disparitätsbereichen innere Beziehungen. So mag etwa die Arbeitsbelastung als zu groß empfunden werden, weil zu lange und $\mathrm{zu}$ hart gearbeitet werden muß, um ein minimales Einkommen zu erreichen. Ferner mag unter zu harter und zu langer notwendiger Arbeit die soziale Anerkennung und die Sinngebung durch die Arbeit leiden.

Drei Ursachen führen u.a. zu Problemdisparitäten, nämlich natürliche Unterschiede, technische Entwicklungen und staatliche (hier agrarpolitische) Eingriffe. Natürliche Unterschiede sind unveränderbar und haben immer und überall zu Disparitäten erwähnter Art geführt. Hier interessieren uns die zwei übrigen Ursachen.

\section{a) Technische Entwicklungen als Ursache von Disparitäten}

Technische Entwicklungen seit dem Zweiten Weltkrieg haben $\mathrm{zu}$ gewaltigen Umwälzungen in der
Arbeitswelt der Industrieländer geführt. Bezüglich der Disparitäten zwischen Berg- und Tallandwirtschaft wirkten sie aus folgenden Gründen vergrößernd: Erstens sind die Maschinenentwicklungen zur Bearbeitung der Felder nicht aus den Verhältnissen im Berggebiet, sondern aus jenen der gewichtigeren Talgebiete (im In- und Ausland) initiiert worden. So fand ein eigentlicher Technologietransfer ins Berggebiet statt. Übermechanisierung vieler Kleinbetriebe ist die Folge, war dies doch der einzige mögliche Weg, um eine Arbeitserleichterung herbeizuführen und um gleichzeitig pro Arbeitskraft mehr zu produzieren. Die Produktivitätsunterschiede zwischen Zonen sind also mit der unterschiedlichen Angepaßtheit der Maschinen größer geworden. Die Produktion pro Arbeitskraft ist stark gestiegen, jedoch in stark unterschiedlichem Ausmaß, während die Preise der Produkte für alle gleichermaßen anstiegen.

Zweitens haben die technischen Entwicklungen zwei Flächenkategorien entstehen lassen, nämlich mechanisch bearbeitbare und mechanisch nicht bearbeitbare. Während im Talgebiet nur kleinere Restgebiete mechanisch nicht bearbeitbar sind, sind dies in höheren Zonen des Berggebiets größere Anteile. In der Regel entsteht so das Brachland, wodurch das Winterfutter zurückgeht und mit diesem die Tierzahl und damit die Anzahl Landwirte. So glauben wir darauf hingewiesen $\mathrm{zu}$ haben, daß die technische Entwicklung als eine weitgehend autonome Ursache für größer gewordene Disparitäten zwischen Tal- und Berggebiet angesehen werden kann. Es wird eine schwierige Aufgabe sein, diesbezüglich eine Trendwende aufzuzeigen.

\section{b) Staatliche Eingriffe als Ursache von Disparitäten}

Vorweg sei der Klarheit wegen betont, daß wir zwei Arten von staatlichen Eingriffen unterscheiden wollen, nämlich eine Kategorie, die Ursachen von größer werdenden Disparitäten sein können, und solchen, die als Folge davon eingeführt wurden, um die Disparitäten abzubauen. Zur ersten Kategorie kann das Landwirtschaftsgesetz vom 3.Oktober 1951 ge-

Peter Rieder, Prof. Dr., Institut für Agrarwirtschaft, ETH-Zentrum, 8092 Zürich 
zählt werden. Seine zwei wichtigsten Artikel des wirtschaftlichen Teiles, nämlich jener über den Außenhandelsschutz und jener über die Gewährung von kostendeckenden Preisen für Inlandprodukte, sind produktmengenorientiert. Mit anderen Worten: Wer als Landwirt garantierte Preise gewährt bekommt, wird in dem Ausmaß davon begünstigt, wieviel er mit seiner Fläche, seinen Tieren und technischen Einrichtungen pro Arbeitskraft produziert. Aus einheitlichen Preisen entstehen wegen oben genannter unterschiedlicher Entwicklungen also stets unterschiedlicherere Einkommen. Jene mit den geringsten Einkommen die Grenzproduzenten - scheiden allmählich aus der Produktion aus, und die Effizienteren vergrößern ihre Marktanteile. Dies ist eine Dynamik, auf der die Marktwirtschaft basiert. Das Landwirtschaftsgesetz hat einerseits diese Dynamik eingeschränkt, indem es Mindestpreise garantierte, also das Gesetz von Angebot und Nachfrage weitgehend ausschaltete. Es basiert aber trotzdem - wie gesagt - auf gesamtschweizerischen Einheitspreisen und wirkte so der Tendenz nach eindeutig disparitätenvergrößernd, und dies statistisch zeigbar bei den Einkommen, und ableitbar bei der Arbeitsbelastung, der sozialen Anerkennung und Sinngebung durch die Arbeit. Folgen und Reaktion blieben nicht aus.

\section{Folgen der Tendenz sich vergrößernder Disparitäten}

Die bisher aufgezeigten Ursachen sich vergrößernder Disparitäten haben zwei Folgen gehabt, die unter dem Aspekt dieses Beitrages besonders hervorzuheben sind, nämlich (a) auf die innerlandwirtschaftliche Arbeitsteilung und (b) auf die Auswirkungen auf die Besiedlung im Berggebiet.

\section{a) Folgen auf die innerlandwirtschaftliche Arbeitsteilung}

Einheitliche garantierte Produktpreise, z. B. für Milch und Fleisch in unterschiedlichen Regionen, führen $\mathrm{zu}$ ebenfalls unterschiedlichen Reaktionen. So ist in den letzten 30 Jahren im Talgebiet und den günstig gelegenen Übergangszonen eine riesige Intensitätssteigerung der landwirtschaftlichen Produktion (mehr ertragssteigernde Hilfsstoffe, höhere Erträge pro Hektare) zu beobachten. Im Berggebiet fanden auf mechanisch bearbeitbaren Flächen ebenfalls Ertragssteigerungen statt. Ertragssteigerungen führen bei garantierten Preisen zu Angebotsausdehnungen. Es entstanden teil- und zeitweise Marktüberschüsse. Bei Absatzbegrenzungen fingen die Talbauern an, ihre Aufzuchtrinder vermehrt selbst aufzuziehen. Die Folge war ein steter Preisdruck auf den Viehmärkten, wo traditionell die Bergbauern den Talbauern ihr Zucht- und Nutzvieh verkaufen. Daraus resultierten Einkommenseinbussen für die Bergbauern. Diese wiederum konnten dies nur kompensieren durch eigene Betriebsvergrößerungen, um mehr Tiere zu verkaufen - oder durch Umsteigen auf «Alternatives» (siehe weiter unten).

Der Arbeitsteilung zwischen Tal- und Berggebiet liegt die Theorie der Komparativen Kosten zugrunde. Angewendet auf unsere Thematik besagt sie folgendes: Absolut betrachtet können im Talgebiet alle Agrarprodukte billiger produziert werden als im Berggebiet, also sowohl Milch wie Zucht- und Nutzvieh. Relativ gesehen ist aber der Kostenunterschied zwischen Tal- und Berggebiet beim Zucht- und Nutzvieh kleiner als bei der Milchproduktion. Bei gegebener Gesamtnachfrage oder der politischen Vorgabe, das Berggebiet sei irgendwie zu bewirtschaften, wäre es volkswirtschaftlich sinnvoll, die Milch im Talgebiet und die Rinder im Berggebiet zu produzieren.

Auf die Tendenz sich vergrößernder Disparitäten zwischen Berg- und Tallandwirtschaft hat die offizielle Agrarpolitik sowohl mit einer Verfeinerung der Zoneneinteilung als auch mit der Einführung von drei Spezialgesetzen zugunsten der Berglandwirtschaft reagiert.

\section{(1) Zur Zoneneinteilung}

Dem Leser dieser Zeilen sind die vielfältigen natürlichen, topographischen und klimatischen Verhältnisse der schweizerischen Landschaft wohl bekannt. Weniger bekannt ist allenfalls, wie diese Unterschiede ihren. agrarpolitischen Niederschlag fanden. Aus einer ursprünglich regional kaum differenzierten agrarpolitischen Behandlung des Tal- und Berggebietes entstanden nach und nach Differenzierungen bis $\mathrm{zu}$ acht verschiedenen Zonen, um Disparitäten abzubauen.

Gewisse Zonen überschneiden sich allerdings, da sie jeweils nur als Grundlage spezifischer Maßnahmen für einzelne Produkte, etwa nur für die Tierhaltung oder nur für den Ackerbau, gelten. Das eigentliche Berggebiet selbst ist zur Zeit in vier Zonen (I bis IV) eingeteilt. Hauptsächlichstes Abgrenzungskriterium zwischen den Zonen ist die Höhenlage, welche die Vegetationsdauer relativ gut erfaßt. Allerdings ist interessant festzustellen, daß die ehemalige Zone III des Berggebietes in eine reduzierte Zone III und eine neue Zone IV aufgeteilt wurde, indem letztere nur noch die steilen Gebiete umfaßt.

Gleichzeitig entstanden auch außerhalb des eigentlichen Berggebietes neue Zonen, so etwa das (ans Berggebiet) angrenzende Zuchtgebiet, die Voralpine Hügelzone, die Übergangszone und eine Erweiterte Übergangszone. Was als eigentliches Talgebiet übrigbleibt, bildet auf der Schweizer Karte einen schmalen Streifen zwischen Genfer- und Bodensee.

\section{(2) Die Spezialgesetze zugunsten der Berglandwirtschaft}

Die offizielle Agrarpolitik hat mit folgenden drei Spezialgesetzen zugunsten der Berglandwirtschaft versucht, die Disparitäten abzubauen: 
- Bundesgesetz über die Förderung des Absatzes von Zucht- und Nutzvieh, von Pferden und Schafwolle, vom 15. Juni 1962 (Absatzgesetz)

- Bundesgesetz über Kostenbeiträge an Viehhalter im Berggebiet und in der voralpinen Hügelzone, vom 28. Juni 1974 (Kostenbeitragsgesetz)

- Bundesgesetz über Bewirtschaftungsbeiträge an die Landwirtschaft mit erschwerten Produktionsbedingungen, vom 14. Dezember 1979 (Flächenbeitragsgesetz)

Alle drei Gesetze dienen grundsätzlich dem Disparitätsabbau. Das Gesetz zur Förderung des Viehabsatzes ermöglicht, Beiträge an Ausmerzaktionen, Entlastungskäufe und an den Viehexport auszurichten. Damit werden die Marktpreise im Inland angehoben. Von dieser Maßnahme profitieren grundsätzlich die Berglandwirte und die italienischen Käufer solcher verbilligter Tiere. Die Belastungen tragen der Bund, aber auch die inländischen Tallandwirte, die wegen diesen Maßnahmen höhere Preise zu bezahlen haben. Höhere Preise bewirken aber wieder einen Rückgang der Nachfrage bzw. einen größeren Anteil eigener Rinderzucht, was seinerseits wieder zu Preisrückgängen auf den Rindermärkten führt. Dieser Zusammenhang soll stellvertretend aufzeigen, wie Interventionen auf Märkten mittelfristig stets Reaktionen hervorrufen, die die beabsichtigte politische Wirkung teilweise wieder aufheben.

Das Gesetz über die Kostenbeiträge ermöglicht, an Bergbauern Geld pro Vieheinheiten direkt auszubezahlen. Hier ist - agrarökonomisch ausgedrückt - die Trennung von Preis- und Einkommenspolitik weitgehend vollzogen worden, aber nur weitgehend, weil wegen Kostenbeiträgen die Berglandwirte tendenziell mehr Tiere halten, weshalb mehr Tiere angeboten werden und in der Folge die Marktpreise wieder sinken. Diesen Senkungseffekt schätzten wir auf rund $20 \%$ des Kostenbeitrages (Rieder, Egger, 1983). Trotzdem sind jedoch die Kostenbeiträge an die Viehhalter im Berggebiet eine Maßnahme, die v. a. Einkommensdisparitäten reduzieren. Um nicht größere Betriebe überproportional zu begünstigen, ist die Beitragsausrichtung auf 15 Grossvieheinheiten pro Betrieb begrenzt.
Das neueste Gesetz ist das Flächenbeitragsgesetz. Dieses gibt dem Bundesrat über einen Rahmenkredit die Möglichkeit, pro Flächeneinheit mit über 18\% Neigung Beiträge auszurichten. Das Gesetz verfolgt zwei Ziele, nämlich Einkommensdisparitäten abzubauen und Brachlandentstehung zu verhindern. Die Einkommenswirkungen sind zwar relativ bescheiden; wir vermuten, daß der Bewirtschaftungseffekt mittelfristig relativ groß sein wird (Michel, 1983).

Der entscheidende Aspekt des Flächenbeitragsgesetzes liegt darin, daß die Beiträge nicht an ein Produkt des Bergbauern, sondern an die bewirtschaftete Fläche gebunden sind. Der Kulturlandschaftserhaltung wird somit gedient, ohne daß sich dadurch das Marktangebot vergrößern muß, was wegen Preissenkungen erneut zu größeren Einkommensdisparitäten führen würde.

\section{b) Folgen für die Besiedlung im Berggebiet}

Die zweite Art der Folgen bezieht sich auf die Entleerung ländlicher Dörfer. Mit der bereits erfolgten Mechanisierung der landwirtschaftlichen Arbeit ist die Zahl der Landwirte in den Dörfern so klein geworden, $\mathrm{da} ß$ die Bauern allein eine zu kleine Gemeinschaft bilden, als daß ein sozio-ökonomisches Gleichgewicht erhalten bleibt. Reine Bauerndörfer haben keine Überlebenschancen, da sie zu klein sind für Dienstleistungsbetriebe, für kulturelle und unterhaltende Anlässe. Treten in einem Dorf viele Nebenerwerbslandwirte auf, ist die Chance etwas besser, aber dennoch und zum Teil aus anderen Gründen, wie Konkurrenz um guten Landwirtschaftsboden - ist die Lebensgemeinschaft gefährdet. Man kann dies am sogenannten «Dorfkonzept) im ländlichen Raum illustrieren:

Dieses Dorfkonzept basiert auf der einfachen Annahme, daß zwischen Wirtschaftszweigen enge gegenseitige Abhängigkeiten bestehen und $\mathrm{da} \beta$ sich andererseits das sozio-kulturelle Leben nur bei einer gewissen Dorfgröße entfalten kann. Aus eingehenden Darstellungen von Beziehungen gelangten wir zur Aussage, daß es eine minimale Dorfgröße gibt, die nicht unterschritten werden darf, wenn das sozioökonomische Gleichgewicht spielen soll. Es ist eindeutig zu beobachten, daß im Kanton Graubünden

Tab. 1 Entwicklung der Bevölkerung nach Gemeindegrößenklassen im Berggebiet (nach IHG) des Kt. Graubünden

\begin{tabular}{|c|c|c|c|c|c|}
\hline \multirow[b]{2}{*}{$\begin{array}{l}\text { Größenklasse } \\
\text { Einwohner }\end{array}$} & \multirow[b]{2}{*}{$\begin{array}{l}\text { Anzahl Gemeinden } \\
\text { pro Größenklasse }\end{array}$} & \multicolumn{2}{|c|}{ Einwohnerzahl } & \multirow{2}{*}{$\begin{array}{c}\text { Geburtenüberschuß } \\
\Delta 1970 / 80 \\
\% \\
\end{array}$} & \multirow{2}{*}{$\begin{array}{c}\text { Wanderungsverlust } \\
\Delta 1970 / 80 \\
\%\end{array}$} \\
\hline & & $\begin{array}{c}1970 \\
\varnothing\end{array}$ & $\begin{array}{c}\Delta 1970 / 80 \\
\%\end{array}$ & & \\
\hline $\begin{array}{r}0-100 \\
100-200 \\
200-500 \\
500-1000 \\
>1000\end{array}$ & $\begin{array}{l}41 \\
46 \\
59 \\
18 \\
22\end{array}$ & $\begin{array}{r}64 \\
140 \\
332 \\
706 \\
1847\end{array}$ & $\begin{array}{l}-9,5 \\
-3,9 \\
-4,2 \\
-1,0 \\
-1,5\end{array}$ & $\begin{array}{l}-3,1 \\
+2,5 \\
+1,7 \\
+4,0 \\
-2,5\end{array}$ & $\begin{array}{l}-6,4 \\
-6,4 \\
-5,9 \\
-5,0 \\
-4,0\end{array}$ \\
\hline
\end{tabular}

Quelle: Eidg. Betriebszählungen 
z. B. die kleinen Gemeinden kleiner werden und die größeren größer. Die Söhne der Landwirte wandern bei zu kleinen Gemeinden nicht mehr aus einkommenspolitischen Gründen ab, sondern aus Gründen fehlender Kommunikation i. w.S. Da bekanntermaBen in der Regel die Landwirtschaft der einzig ortsgebundene Wirtschaftszweig ist, wird erst eine Abwanderung aus ihr als Verlust empfunden, obwohl in den ländlichen Dörfern diese Tendenz weit früher eingesetzt hat, vielleicht dann, als das Handwerk den Platz für fabrikmäßig hergestellte Produkte räumen mußte.

Unsere nachfolgenden Ausführungen gehen nun sowohl von den aufgezeigten Folgen innerhalb der Landwirtschaft selbst als auch von der Annahme, dass längerfristig die Landwirtschaft als ortsgebundener Wirtschaftszweig im abgelegenen ländlichen Raum von der Existenz eines Dorfes im sozio-ökonomischen Gleichgewicht abhängt, aus.

\section{Neue Möglichkeiten des Disparitätenabbaus zwischen Tal- und Berglandwirten sowie zwischen Landwirten und Nichtlandwirten im Berggebiet}

Neue Versuche zur Verringerung der Disparitäten setzten Vorstellungen über Veränderungen in den Strukturen in der Berg- und Tallandwirtschaft voraus. Diese Veränderungen haben sowohl der Besiedlung als auch der Individualität der Einzelbauern zu dienen. Der Begriff «Strukturerhaltung» als solcher ist zu eng. Es sind Werte zu erhalten, auf die Strukturen auszurichten sind. Der Bergbauer muß für sich die von ihm produzierten Güter als Werte erleben können. Seine Leistungen dürfen nicht nur subventionierte Überschußprodukte sein, von denen die Talbauern kostengünstiger auch bereits Überschüsse produzieren (RIEDER, 1984).

Die neuen Möglichkeiten zum Abbau der Disparitäten wollen wir nach drei Arten ordnen, nämlich a) mehr Effizienz bei bestehenden Maßnahmen, b) Alternativen fördern und c) Kleinbetriebe existenzfähig machen.

\section{a) Mehr Effizienz bei bestehenden Maßnahmen}

Wie weiter oben erwähnt, wurden in den letzten zwanzig Jahren eine Anzahl von Maßnahmen zugunsten des Berggebietes ergriffen. Grundsätzlich dienten alle dem Disparitätsabbau zwischen Tal- und Berggebiet bzw. zwischen Landwirten und übrigen Berufstätigen. Es ist hier nicht der Platz, die einzelnen Maßnahmen auf ihre Wirksamkeit hin zu überprüfen. Jedoch seien einige Bemerkungen grundsätzlicher Art angebracht. Ein erster kritischer Aspekt bezieht sich auf die Möglichkeit von kontraproduktiver Wirkung einzelner Maßnahmen. Hierzu einige Beispiele: (1) Wie schon erwähnt, ist es erwiesen, daß hohe Export- beiträge zu inländischen Preiserhöhungen führen und diese ihrerseits zu Nachfragerückgängen seitens der Tallandwirte führen. Hier wäre also eine Art optimale Kombination zwischen den verschiedenen absatzpolitischen Maßnahmen zu suchen. (2) Bisherige Preisverhältnisse zwischen Milch einerseits und anderen Produkten bewirkten, daß z. B. die Schafhaltung nach einem Aufschwung in den siebziger Jahren wieder zurückging, weil Milch stets die bessere betriebswirtschaftliche Alternative war, obwohl Schafe sich für Berggebiete eignen und hiefür auch keine Absatzprobleme bestehen. (3) Strukturpolitische Hilfen (Subventionen für Güterzusammenlegungen, für Einzelwegbauten und Hochbauten) sind weniger ausgebaut worden als die oben erwähnten preis- und absatzpolitischen Maßnahmen. Dieses Gleichgewicht wäre zu erforschen und zu praktizieren. In der politischen Praxis genießen bei Finanzknappheit jedoch die einkommensmäßig kurzfristig wirkenden Maßnahmen stets den Vorzug. Beiträge an Meliorationswerke sind politisch leichter zu kürzen, als Milchpreise zu senken. So gäbe es zahlreiche weitere kritische Aspekte zu erwähnen, die theoretisch verbesserungsfähig wären.

\section{b) Alternativen fördern}

Hier sollen also einige Ausführungen zur Rolle von alternativen Produktionsmöglichkeiten im Berggebiet erfolgen. Den Ausgangspunkt bildet folgende Aussage: Wird bei der Produktion 5 Prozent Konventionelles durch 5 Prozent Alternatives ersetzt, so geht es der Berglandwirtschaft bei gleichem öffentlichem Aufwand nachher besser als vorher.

Die Schweiz importiert eine große Palette mengenmäBig unbedeutender Produkte, die in der Schweiz auch produziert werden könnten, vermutlich zwar teurer als zum Importpreis. Volkswirtschaftlich profitieren wir dabei von billigen Importen, zum Beispiel von Wild, Schaffleisch, Kaninchen, Ziegenfleisch, Eiern usw. Anderseits verbilligen wir Exporte, die von Flächen stammen, die auch für Alternatives geeignet wären. Da wir mit dem Konventionellen auf übersättigte Absatzmärkte müssen, kostet uns eine Hektare mehr, als wenn wir alternativen Produkten einen entsprechenden Absatzschutz auf preiselastischeren Märkten gäben.

Ein weiterer Aspekt: Alternatives wird absolut stets in sehr begrenztem Ausmaß bleiben. Sein Anteil reduziert indirekt aber das Angebot an Konventionellem; es trägt also zu Marktentlastungen bei den Hauptprodukten bei. Darum sollten eigentlich alle Konventionellen froh sein über jeden, der Alternatives ausprobiert.

Ein dritter Aspekt: Siedlungspolitisch ist Alternatives sehr erwünscht, weil es in der Regel beschäftigungswirksamer als extensive Rauhfutterproduktion ist. Braucht das Berggebiet doch Familien, die in funktionsfähigen Dorfgemeinschaften leben. Also müßte 
eigentlich alles unternommen werden, um die arbeitsintensive Produktion - ob alternativ oder konventionell - in der Berglandwirtschaft zu erhalten und zu fördern.

\section{c) Kleinbetriebe existenzfähig machen}

Die letzten Zeilen des vorigen Abschnittes rufen nach einer Neuausrichtung der landwirtschaftlichen Betriebsstrukturen. Wie hätten diese auszusehen, bzw. wie müßte die heutige Bergbauernpolitik geändert werden, wenn der Erhaltung funktionsfähiger Dorfgemeinschaft höchste Priorität eingeräumt würde?

Die Entwicklungen der letzten dreißig Jahre waren im Berggebiet geprägt durch eine bevorzugte Preis- und Absatzpolitik für Produkte aus der Rindviehhaltung einerseits und durch die Strukturpolitik, die auf relativ großflächige Graswirtschaftsbetriebe mit großen Rindviehbeständen ausgerichtet war. Diese Politik ist aus der Interessenlage der betroffenen Landwirte und ihrer agrarpolitischen Vertreter verständlich. Denn man ermöglichte dadurch eine starke Mechanisierung der landwirtschaftlichen Arbeit und eine beachtliche Steigerung ihrer Arbeitsproduktivität. Gleichzeitig war der Sog der übrigen Wirtschaft auf Arbeitskräfte so groß, daß selbst in der Berglandwirtschaft da und dort ein Arbeitskräftemangel auftrat.

Die heutige Folge dieser Politik als auch der allgemeinen Wirtschaftslage ist nun, daß zu wenig Familien in den Dörfern leben, um die Besiedlung aufrechtzuerhalten. Soll also die Besiedlung gefördert werden, braucht es hiefür für einzelne Familien neue Anreize und tragende wirtschaftliche Rahmenbedingungen. Mit anderen Worten: es sind Voraussetzungen zu schaffen, daß kleinere landwirtschaftliche Betriebe existieren können. Wie aber kann dies geschehen?

Wir haben uns in jüngster Zeit intensiv mit dieser sehr schwierigen Frage beschäftigt und werden es auch in Zukunft tun. Es ist unseres Erachtens eine Art Zukunftsforschung, die nicht nur die Berggemeinden selbst, sondern die ganze schweizerische Öffentlichkeit angeht.

Unsere diesbezügliche Arbeit gliedert sich in drei Phasen, nämlich (1) in eine empirische Erfassung von bestehenden Alternativbetrieben, (2) in eine planerische Erarbeitung von existenzfähigen Kleinbetrieben und (3) in Agrarpolitische Konsequenzen.

\section{(1) Empirische Erfassung von bestehenden Alternativbetrieben}

Um uns ein Bild über existierende Alternativbetriebe zu verschaffen, haben wir empirische Untersuchungen durchgeführt, nämlich über «Ziegenhaltung, sowie Herstellung und Absatz von Bündner Ziegenkäse» (BRÄM et al. 1981) und über "Produktionsalternativen in der Berglandwirtschaft) (DECURTINS et al. 1982). Zum Verständnis der nachfolgenden agrarpolitischen Konsequenzen geben wir hier die Zusammenfassung aus obigen Arbeiten wieder:
Um Produktionsalternativen für die Berglandwirtschaft beurteilen zu können, ist nach der Wirtschaftlichkeit, der Schaffung oder Erhaltung von Arbeitsplätzen sowie nach der Bildung von Einkommen zu fragen. Unter Berücksichtigung dieser Kriterien stellen die folgenden Produktionsrichtungen mögliche Alternativen für die Berglandwirtschaft dar: Damwild, Milchschafe, Ziegen (zur Käseproduktion), Beeren, Gemüse, "Biologische Produkte» und Nahrungsmittelspezialitäten. Sie zeichnen sich durch ein relevantes Marktvolumen und relativ gute Preise aus. Verbesserungsfähig ist aber in einigen Fällen die Wirtschaftlichkeit und die Technologie. Mit größeren Einschränkungen sind auch folgende Betriebszweige als alternative Produktionsmöglichkeiten denkbar: Rotwild, Gänse, Truten, Bienen, Kräuter, Tabak und Pilze.

Die Schwachstellen bei diesen sind recht unterschiedlich. Beim Rotwild ist es die Öffentlichkeit, die sich gegen diese Produktionsform stellt. Die Gänse- und Trutenhaltung leiden vor allem unter der fehlenden Wirtschaftlichkeit; hinzu kommt die größtenteils bodenunabhängige Futterbeschaffung. Die Imkerei hat mit der starken Auslandkonkurrenz zu kämpfen. Bei den Kräutern erlaubt das relativ bescheidene Absatzvolumen lediglich geringe Anbauflächen. Der Kräuteranbau ist auch durch klimatische Anforderungen begrenzt. Bei den Pilzen fehlt noch die angepaßte Technologie. Das vorliegende Ergebnis zeigt, da $\beta$ die Produktionsalternativen für unsere Berglandwirtschaft vor allem im Bereiche der arbeitsintensiven Tierhaltung gesucht werden müssen. Tierische Erzeugnisse lassen sich überdies leichter im eigenen Betrieb weiterverarbeiten, was zusätzliches Einkommen ermöglicht. Neben dem Gesagten ist die Persönlichkeit des Betriebsleiters wichtig. Der idealtypische Alternativbauer zeichnet sich durch viel Eigeninitiative, große Risikofreudigkeit, eine "Pröblernatur», hohe geistige Beweglichkeit, Ausdauer und ein ausgeprägtes soziales Durchsetzungsvermögen aus. Die in unserer Untersuchung gewonnenen Erkenntnisse lassen keinen großen Optimismus aufkommen; aber ebensowenig scheint Resignation am Platze. Die Bestrebungen zur Realisierung von landwirtschaftlichen Produktionsalternativen müssen zukünftig vermehrt auf die Bedürfnisse der Absatzseite ausgerichtet werden. Es wären vermehrt Kontakte mit den an einer Sortimentserweiterung sehr interessierten Grossisten aufzunehmen, um marktgerechte Güter in relevanten Mengen zu produzieren. Zudem ist die Forschung der diversen Technologien $\mathrm{zu}$ intensivieren. Interessant scheint dies vor allem im Bereiche des biologischen Landbaus, wo im Trend zu gesunder Ernährung ein zwar komplizierter, aber doch unterversorgter Markt abzudecken ist.

\section{(2) Planerische Erarbeitung von Kleinbetrieben}

Bevor ein Landwirt einen Betrieb neu aufbaut oder eine große Umstellung vornimmt, muß geplant werden. Je komplizierter die Probleme sind, um so 
eher lohnt es sich, dies mit wissenschaftlichen Methoden zu tun. Für Kleinbetriebe im Berggebiet existenzsichernde Betriebsformen zu suchen ist nicht einfach, so daß wir wissenschaftliche Methoden (Lineare Optimierungsmodelle) anwendeten. Auf einfache Art ausgedrückt arbeiten diese Methoden folgendermaßen: Man gibt in einem Modell alle physisch möglichen Produktionsalternativen, also von der konventionellen Rindviehhaltung bis zur Erdbeerenproduktion, zur freien Auswahl vor, bewertet diese mit Aufwänden, Kosten und Preisen und sucht unter Vorgabe verschieden großer Flächen das betriebswirtschaftliche Optimum. Als Resultat erhält man verschiedene Kombinationen von Betriebszweigen, Tierarten, Flächennutzungen und das Einkommen für die mitarbeitenden Familienmitglieder.

Die konkreten Planungen führten wir für die Fläche und unter den natürlichen Bedingungen des Beneficiums Rumein (Degen/Igels) durch. Wir können unsere Ergebnisse, soweit diese von Allgemeininteresse sind, folgendermaßen charakterisieren:
Arbeiten dieser Art haben noch einen provisorischen Charakter, da sie planerischer und nicht empirischer Art sind. Unseres Erachtens weisen sie aber doch in eine Richtung, die aufzeigt, daß unter einigen agrarpolitischen Veränderungen existenzfähige Betriebe dieser Art entstehen könnten. Darauf kommen wir im nächsten Abschnitt zurück.

\section{(3) Agrarpolitische Konsequenzen}

Aus den dargestellten Problemen und Hinweisen auf Möglichkeiten einer Neuausrichtung einer Anzahl Betriebe im Berggebiet ergeben sich agrarpolitische Konsequenzen, die hier gleichzeitig als Denkanstöße und Schlußfolgerungen aufzufassen sind. Dabei haben wir die Bergtäler im Sinne, die etwa mittelmäßige natürliche Verhältnisse aufweisen. In den ungünstigsten Verhältnissen wird es kaum Alternativen zur traditionellen Rindviehhaltung geben.

Zur Strukturpolitik kann gefolgert werden, daß sie gegenüber heute in verstärktem Maße den örtlichen Verhältnissen flexibel angepaßt werden muß. Bei

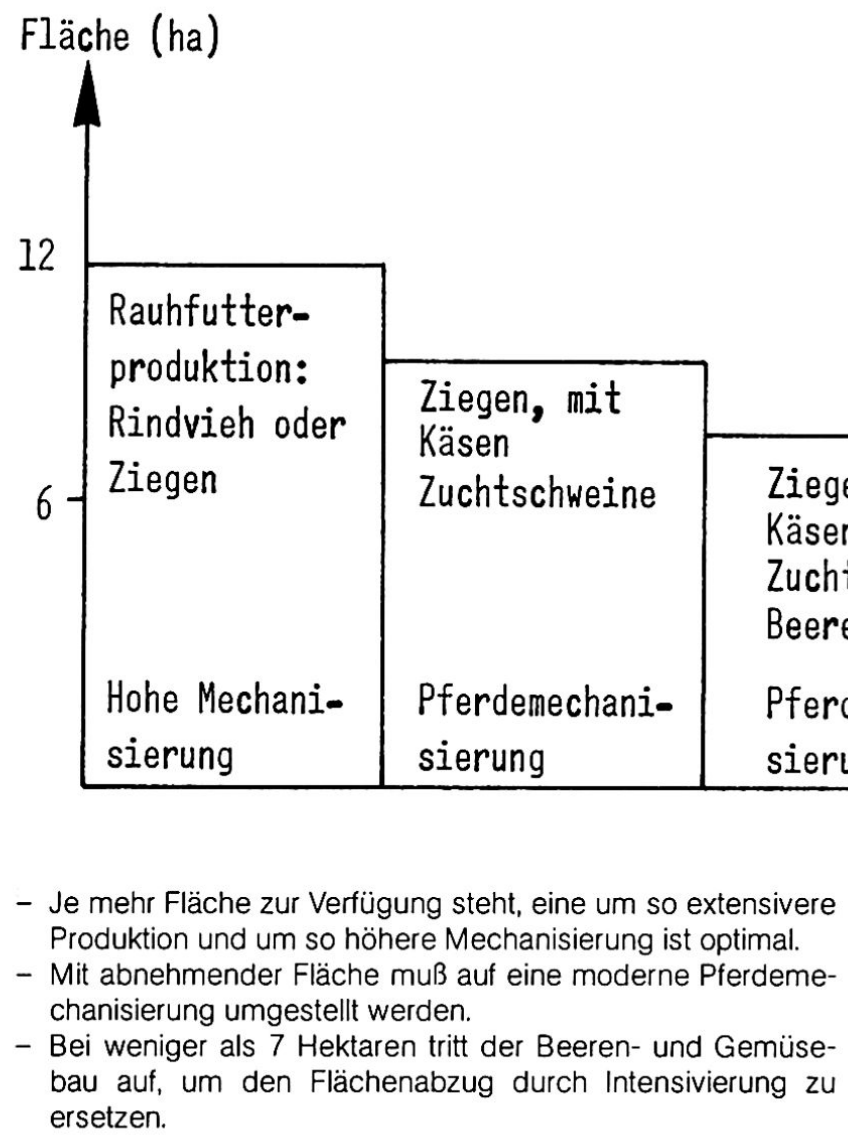

- Bei rund 5 bis 6 Hektaren werden einige Kühe und Zuchtschweine und eine halbe Hektare Gemüse optimal. Gegenüber den großflächigen Varianten ist ersichtlich, daß eine halbe Hektare Gemüse etwa 7 ha Rauhfutterfläche ersetzt.

- Je kleiner der Betrieb, desto nötiger sind arbeitsintensivere Betriebszweige und betriebseigene Verarbeitung von Produkten.

Abb.1 Schematische Darstellung von Planungsergebnissen in Abhängigkeit der vorgegebenen Fläche 
kleinflächigen Betrieben sind bei vorliegenden Betriebsplänen weniger restriktive Bedingungen an den Erhalt von Investitionskrediten und Subventionen zu knüpfen. Bei Güterzusammenlegungen müssen ihre Flächenansprüche qualitativ auf die nötige intensivere Nutzung Rücksicht nehmen. Daneben wird es viele großflächige Betriebe geben müssen, die andere spezifische Bedürfnisse haben. Letztere leisten insbesondere einen öffentlichen Beitrag an die Kulturlandschaftserhaltung im Berggebiet und sind nicht weniger wichtig als die kleinen Betriebe.

Von besonders großer Bedeutung sind allfällige Veränderungen in der Agrarpolitik. Wie schon erwähnt genießen heute die Produkte aus der Rindviehhaltung die gesichertsten und relativ höchsten Preise. Dies soll nicht abgeändert werden, wohl aber ist es eine notwendige Voraussetzung, die Preisverhältnisse für übrige Produkte entsprechend zu sichern und anzuheben, wenn Umstellungen in spürbarem Ausmaß erfolgen sollten. Zum Beispiel wären im Verhältnis zu Milch- und Rinderpreisen Preisparitäten für Schafe, Ziegenprodukte, Gemüse, Beeren und ähnliches einzuführen. Man könnte sich hierbei eine Art Ausgleichszahlungen vorstellen, indem Landwirten die Differenz zwischen Markt- und Sollpreisen ausbezahlt würde. Aber es wären auch Verträge zwischen Abnehmern und kantonalen Stellen denkbar oder gar Übernahmemärkte wie beim Großvieh. Mehr Administration als beim Großvieh entstünde nicht. Wichtiger als genannte Einrichtungen wäre jedoch ein spezifischer Grenzschutz für einige Arten von im Berggebiet produzierbaren Produkten. Während für Kuhmilch ein perfekter Grenzschutz besteht, ist zu beobachten, daß die wenigen Ziegen- und Schafkäsehersteller in der Schweiz neben den viel größeren und billigeren Importen kaum zu bestehen vermögen. Das gleiche gilt für die Fleischlämmer, würden sich doch die Berggebiete vielerorts für Schafhaltung eignen.

Koppeln wir diese Konsequenzen zurück zum Thema Disparitätenabbau, so ist damit ein Weg gezeigt, wie der wirtschaftliche Freiraum für Berglandwirte geöffnet würde. Die Betriebsstrukturen würden wieder vielfältiger, es könnte wieder mehr für die eigene Selbstversorgung produziert werden. Traditionelle überlastete Märkte würden - um es nochmals zu betonen - nach und nach entlastet. Es würden sich also Umlagerungen einstellen, die insgesamt $\mathrm{zu}$ einer Aufwertung der Berglandwirtschaft führen würden. Damit wären Weichen für einen langfristigen Disparitätenabbau gestellt.

\section{Summary}

Regional Disparities between Plane and Mountain Agriculture and Means for their Diminishing

This article deals with the disparities between mountain and plane agriculture in Switzerland. A first part shows the causes. It states that beside natural conditions as well the development of the specific technology as well the federal agricultural laws lead to increasing disparities. The consequences are discussed, too. The federal agricultural policy responded with three special laws in favour of the mountain agriculture. The character of these laws are also discussed. The problems are not yet solved, specifically it can be observed, that the small villages get smaller and the big are growing.

The article discusses a number of possibilities to diminish the disparities within the Swiss agriculture: these are (a) more efficiency of the already existing measures, (b) incentives for «new» products and (c) measures, which lead to conditions, that small farms could reach a full economical existence. This again would increase the number of families in the small mountain villages. Results of planning and field studies are presented.

\section{Literatur}

BRÄM, E., DECURTINS, M. C., RIEDER, P. (1981): Ziegenhaltung, sowie Herstellung und Absatz von Bündner Ziegenkäse. Agrarwirtschaftliche Studie, Nr. 17, ETH Zürich.

DECURTINS, M. C., KLEE, T., RIEDER, P. (1982): Produktionsalternativen in der Berglandwirtschaft. Arbeitsberichte NFP "Regionalprobleme in der Schweiz", Bern.

MICHEL, A. (1983): Erste Auswirkungen der 1980 eingeführten Flächenbeiträge für die Berglandwirtschaft. Diplomarbeit ETH, Zürich.

RIEDER, P., EGGER, U. (1983): Agrarmärkte, vdf, Zürich.

RIEDER, P. (1984): Werte und Wertschöpfung der Berglandwirtschaft. In: Brugger, E.A. et al. (Hrg.) (1984): Umbruch im Berggebiet, Haupt, Bern. 